DIVISION OF THE HUMANITIES AND SOCIAL SCIENCES

CALIFORNIA INSTITUTE OF TECHNOLOGY

PASADENA, CALIFORNIA 91125

DESIGN IMPROVED PARIMUTUEL-TYPE INFORMATION

AGGREGATION MECHANISMS: INACCURACIES AND THE LONG-SHOT BIAS AS DISEQUILIBRIUM PHENOMENA

Boris S. Axelrod

California Institute of Technology

Benjamin J. Kulick

California Institute of Technology

Charles R. Plott

California Institute of Technology

Kevin A. Roust

University of California, San Diego

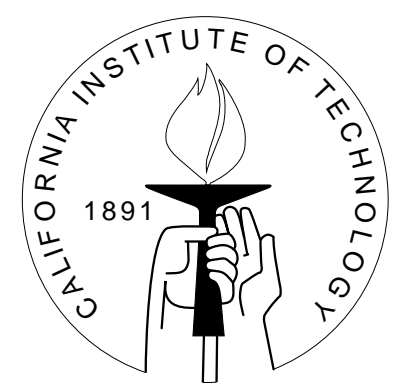

SOCIAL SCIENCE WORKING PAPER 1268 


\title{
Design Improved Parimutuel-type Information Aggregation Mechanisms: Inaccuracies and the Long-Shot Bias as Disequilibrium Phenomena
}

Boris S. Axelrod Benjamin J. Kulick Charles R. Plott Kevin A. Roust

\begin{abstract}
Information Aggregation Mechanisms (IAMs) based on parimutuel-type betting systems can aggregate information from complex environments. However, the performance of previously studied systems leaves something to be desired due to possible bluffing, strategic timing of decisions and a so called long shot bias. This paper demonstrates that two modifications of parimutuel systems improve information aggregation performance by removing disinformation due to strategic behavior and by removing misleading disequilibrium behavior. The experiments also demonstrate that the so called long shot bias results from disequilibrium behavior as opposed to having roots in the psychology of the individuals.
\end{abstract}




\title{
Design Improved Parimutuel-type Information Aggregation Mechanisms: Inaccuracies and the Long- Shot Bias as Disequilibrium Phenomena
}

\author{
Boris S. Axelrod, Benjamin J. Kulick, Charles R. Plott, Kevin A. Roust
}

This paper ${ }^{1}$ explores improvements in the information aggregation capacity of a parimutuel based information aggregation mechanism first studied by Plott, Wit, and Yang (2003). The overall conclusion of Plott, Wit, and Yang (PWY) is that odds produced by the parimutuel system can be translated into probabilities that carry much of the information among agents. That is, the bets placed by agents capture and aggregate information known to exist in individual participants even though the individuals have no particular incentive to reveal their information. The focus of this paper is on changes in the institutional and incentive features of the mechanism that improve the process.

That markets have the capacity to aggregate information was first discovered by Plott and Sunder $(1982,1988)$. This discovery lead to the design and deployment of the first information aggregation mechanism created explicitly for the collection of diverse opinions found in field applications (Chen and Plott 2002, Plott 2000). Those successes led to questions about the existence of completely different forms of information aggregation mechanisms and to the experimental work of PWY. This paper is a natural continuation of a line of investigation of the institutions and designs that lead to the best performance. An additional body of literature has developed, typically under the heading of "prediction markets". While there is a clear relationship, that literature is not focused on information aggregation and will not be addressed here. There are also IAMs that deal with much more complex interactions, specialized instruments and computerized reporting, a discussion of which would also remove the focus from the points addressed here that deal with parimutuel betting-type mechanism.

While PWY concluded that the parimutuel process they studied did indeed result in aggregated information, they noticed clear deficiencies. First, strategic, "last second" delays in the placing of bets tended to reduce the information to which the system was exposed. Secondly, aspects of bluffing seem to interact with delays creating much disequilibrium behavior. In addition, PWY observed a systematic bias in the information

\footnotetext{
${ }^{1}$ The financial support of the National Science Foundation is gratefully acknowledged. The comments of David Grether, Colin Camerer, and Robert Sherman have been very helpful.
} 
content of their data similar to features of data from betting processes. Rare events are predicted with probabilities that are too high. Similar patterns are frequently observed in horse races and other parimutuel betting systems and have become recognized as a "long shot bias" (see Thaler and Ziemba, 1988 for an introduction of the concept in the context of other betting systems). From the point of view of information aggregation mechanisms and their design, the implications of the bias depend on the reason for its existence.

Two different theories compete as explanations of the long shot bias as observed by PWY. One rests on a conjectured psychological principle that individuals tend to "overweigh" events with small probabilities. Thus, the psychological theory of the bias suggests the existence of fundamental limitations on the ability of any information aggregation device to gather the information that is located in the experiences of people. The theory suggests that because of human limitations the information to which individuals have been exposed cannot be retrieved, regardless of the mechanism. If that is the case, then the design of information aggregation mechanisms faces important constraints.

A second theory about the long shot bias is more optimistic about the prospects for the design of information aggregation mechanisms. This second theory suggests that the long shot bias is a consequence of disequilibrium behavior. In a parimutuel system, once a bet is placed it cannot be undone ${ }^{2}$. And, since fluctuation of odds during the betting process could cause almost any pattern of bets to be reasonable at one time or another, the nature of the long shot bias would simply be a consequence of the particular dynamics.

Our attention thus shifts to disequilibrium choices and strategies that foster disequilibria. If the disequilibrium theory of the long shot bias is correct, then a step in the right direction would be to change the nature of the mechanism so the disequilibria are removed. Two substantial sources of disequilibria suggest themselves immediately. The first is strategic delay of choices, which follows from theory and is clearly demonstrated by PWY. By delaying actions and observing the actions of others before deciding, an agent not only learns from the decisions of others but also masks his/her own information that might benefit opponents. The second source of disequilibria is a consequence of information aggregation itself. A sequential updating is possible as information is passed back and forth, being updated at each iteration based upon the just received update. If cut short, prior to approaching any asymptote the system will not have aggregated all of the information that exists to be aggregated.

In this paper, two institutional changes are made to the PWY mechanism. First, a cost of delay is imposed. In a sense, a tax on waiting is established so those who place bets earlier receive a benefit for doing so. The feature is implemented as the increasing price

\footnotetext{
2 There are parimutuel systems in which bets can be cancelled but cancellation involves inherent costs. See Camerer (1998).
} 
of tickets to replace a fixed size bet and thus the term “increasing price parimutuel”. The second is a simple replication of the exercise. After all bets are made, the betting windows are closed and the odds are published but with no winner announced, a new round of betting begins with an independent "pot" for winners. In this new round of betting, all information (except the winning strategy) emerging from the first round of betting is known. Thus, the second round does not have the disequilibrium elements of the first round. In a sense, it is much like the idea of a "tatonnement" invented to remove the theoretical problems of disequilibrium trades in markets.

The basic result is that the cost of delay speeds the information aggregation process and improves the accuracy of the mechanism. In addition, these institutional innovations successfully remove the long shot bias thereby suggesting that disequilibrium betting and not the psychology of the individuals is the cause of the bias. Information aggregation was further improved by the repeated round of betting.

\section{Structure of The Increasing-Price Parimutuel IAM}

Fundamentally, each subject was assigned the task of identifying the true state of the world, which was drawn from the set $\{A, B, C, D, E, F\}$. Each subject was provided with three independent signals as to this state and then the agents were allowed to interact in a parimutuel market setting. Their purchases in the market and the true state of the world then determined their earnings.

A particular experiment consisted of a number of periods. Each period contained four major phases:

1. Random selection of "true state" for the period (revealed only to experimenter).

2. Distribution of private information about the "true state" to each subject.

3. Markets open for purchases and then close two minutes later. In the repeated periods, the windows were opened again and a second betting began for a separate pot of money.

4. "True state" is publicly announced and earnings information is distributed.

\section{Institutional Framework of the Parimutuel IAM}

Much of the institutional structure employed here is based on the work of Plott, Wit, and Yang (2003), who considered a parimutuel mechanism based loosely on racetrack betting systems. For ease of communication, we will refer to such processes as "markets" even though there is little market activity other than ticket sales. In the experiments, each subject was provided with a quantity of experimental money ("francs", converted to U. S. dollars at the end of the experiment) that could be used to purchase "tickets" corresponding to the six possible states. The experimenter would sell these tickets from the opening of the market until a designated closing time. 
After the market closed, earnings would be calculated based on individual ticket purchases and the "true state" in the following manner. First, the winnings for each "true state" ticket would be calculated:

Total Revenue from Ticket Sales

$$
\text { Per Ticket Winnings }=\frac{\text { plus House Bonus }}{\text { Total Number of Winning Tickets }}
$$

Then an individual earns:

Number of Winning Tickets * Per Ticket Winnings

+ Unspent Experimental Money - Loan

As a period progressed, the total tickets sold and "Per Ticket Winnings" (conditional on the market ending instantly and the given state winning) were reported publicly for each state.

The increasing-price parimutuel mechanisms studied here have two prominent additional features. First, the closing time was fixed. Secondly, the ticket prices increased while the market was open. In particular, the market was open for two minutes and the ticket price would increase at a rate of one franc per second from an initial 90 francs per ticket. The time remaining before closing and the current price was reported publicly at all times. This feature justifies the use of the term "increasing-price parimutuel markets".

The second feature was the repeating of a period, which was developed to remove some of the disequilibrium elements and thus test the long shot bias against the disequilibrium hypothesis. Some periods were (public) repeats of the previous period with the exact same true state and private information. The earnings and true state of the first period could not be revealed until after both periods were completed. These periods will generally be referred to as "repeated increasing-price parimutuel markets". To the extent that the first period reflected equilibration the second period reflected no equilibration and if the long shot bias was due to disequilibria as opposed to the psychology of the individuals, then the long shot bias should not be observed in the repeated increasingprice parimutuels.

This leads to the rounds in the described experiments being classified into three separate categories:

- "Non-Repeat" Rounds - a new true state and new private information are generated before these rounds and earnings information is reported immediately after the round.

- "First of a Repeat Pair" Rounds - a new true state and new private information are generated before these rounds, but earnings information is not immediately reported after the round.

- "Second of a Repeat Pair" Rounds - the same private information is re-issued and earnings for both this round and the previous round are reported immediately after the round.

Much of the structure of these experiments is adapted from Plott, Wit, and Yang (2003) and the major differences should be emphasized. The PWY mechanism utilized a 
random closing time while the mechanism studied here used a fixed closing time. PWY used a constant ticket price as opposed to the increasing ticket price used here. PWY had no repeated periods by contrast to the experiments reported here, so each period of the PWY involved its own equilibration process.

\section{Information Structure}

The most direct connection is that the information structure used in these experiments exactly matches the second treatment of Plott, Wit, and Yang (their Probabilistic Information Condition, "PIC").

Each period would start with a computer selecting one of the six possible states from an equiprobable distribution (1/6 probability for each state). This selection would be indicated to the experimenter and would be used as the "true state" for that period.

The period would continue with the computer generating three independent signals of the state for each subject. These signals correspond directly to the possible states of the world and are drawn from $\{\mathrm{A}, \mathrm{B}, \mathrm{C}, \mathrm{D}, \mathrm{E}, \mathrm{F}\}$. The signals were generated with a $1 / 3$ probability of receiving a correct signal (signal matches true state) and a 2/15 probability for each incorrect signal (signal is different from the true state). If the true state were $\mathrm{A}$, then it would be as if the signals were drawn independently (with replacement) from an urn containing fifteen balls labeled as follows:
A A A A A
B B
C C
D D
E $\quad E \quad F \quad F$

Occasionally these private signals will be grouped into "Information Types", referring to the number of matching signals that a subject received. A subject at "InfoType 3" received three matching signals (e.g. AAA or BBB,etc.). A subject at "InfoType 2" received exactly two matching signals (e.g., AAB). A subject at "InfoType 1" received three different signals (e.g., ABC).

Since each subject receives three independent draws, their private information has a multinomial distribution with six possible outcomes and three trials with the probabilities identified above. This distribution has the following joint probability mass function:

$$
\begin{aligned}
& f\left(x_{A}, \ldots, x_{F} \mid \text { True State }=S\right)=\frac{m !}{x_{A} ! \cdots x_{F} !} p_{C}^{x_{S}} p_{N}^{x_{T}-x_{s}} \\
& x_{i}=\text { Number of signals matching } \mathrm{i} \\
& x_{T}=\sum_{i=A}^{F} x_{i}=\text { Total number of signals } \\
& m=3 p_{C}=\frac{1}{3} p_{N}=\frac{2}{15}
\end{aligned}
$$

Bayes' Law can then be used in the standard way to identify the posterior probability distribution over the states given the private signal. 


$$
P(S \mid x)=\frac{f(x \mid S) P(S)}{\sum_{i=A}^{F} f(x \mid i) P(i)}=\frac{f(x \mid S)}{\sum_{i=A}^{F} f(x \mid i)}
$$

(since $P(i)=\frac{1}{6}$ for all states)

These posterior probabilities can be easily summarized in a simple table:

\begin{tabular}{|c|c|c|c|c|}
\hline \multicolumn{5}{|c|}{ Posterior probability distribution across states given private information } \\
\hline & $\begin{array}{c}\text { most } \\
\text { frequent } \\
\text { letter (A) }\end{array}$ & $\begin{array}{c}\text { second most } \\
\text { frequent } \\
\text { letter (B) }\end{array}$ & $\begin{array}{c}\text { third most } \\
\text { frequent } \\
\text { letter (C) }\end{array}$ & $\begin{array}{c}\text { each of the } \\
\text { other letters } \\
\text { (D, E, F) }\end{array}$ \\
\hline $\begin{array}{c}\text { all three draws are the } \\
\text { same letter (AAA) }\end{array}$ & $75.8 \%$ & $4.8 \%$ & $4.8 \%$ & $4.8 \%$ \\
\hline $\begin{array}{c}\text { two of the three draws are } \\
\text { the same letter (AAB) }\end{array}$ & $49.0 \%$ & $19.6 \%$ & $7.8 \%$ & $7.8 \%$ \\
\hline $\begin{array}{c}\text { all three draws are different } \\
\text { letters (ABC) }\end{array}$ & $23.8 \%$ & $23.8 \%$ & $23.8 \%$ & $9.5 \%$ \\
\hline
\end{tabular}

\section{Experimental Design and Procedures}

Experiment parameters are as follows:

\begin{tabular}{|c|c|c|c|c|c|c|c|}
\hline Parameters & 010215 & 010222 & 010315 & 010419 & $\begin{array}{c}010426 \\
2100\end{array}$ & $\begin{array}{c}010426 \\
2230\end{array}$ & 010510 \\
\hline Subjects & 18 & 10 & 9 & 10 & 10 & 10 & 19 \\
\hline Endowment & 9000 & 1800 & 1800 & 1800 & 1800 & 1800 & 1800 \\
\hline Loan & 13500 & 2700 & 2700 & 2700 & 2700 & 2700 & 2700 \\
\hline House Bonus & 45000 & 9000 & 9000 & 9000 & 9000 & 9000 & 9000 \\
\hline $\begin{array}{l}\text { Practice } \\
\text { Round \#s }\end{array}$ & 0 & 0 & 0 & 0 & 0 & 0 & 0 \\
\hline $\begin{array}{l}\text { Non-Repeat } \\
\text { Round \#s }\end{array}$ & $1-4$ & $1-4$ & $\begin{array}{c}1-4 \\
17-20 \\
\end{array}$ & $\begin{array}{c}1-4 \\
17-20 \\
\end{array}$ & $\begin{array}{c}1-4 \\
17-20 \\
\end{array}$ & $\begin{array}{c}1-4 \\
17-20 \\
\end{array}$ & $\begin{array}{c}1-4 \\
17-20 \\
\end{array}$ \\
\hline $\begin{array}{l}\text { Repeat } \\
\text { Round \#s }\end{array}$ & $5-16$ & $5-16$ & $5-16$ & 5-16 & $5-16$ & $5-16$ & 5-16 \\
\hline $\begin{array}{l}\text { Own Money } \\
\text { Round \#s }\end{array}$ & $17-20$ & $17-20$ & None & None & None & None & None \\
\hline $\begin{array}{l}\text { Exchange } \\
\text { Rate francs/\$ }\end{array}$ & 4500 & 900 & 900 & 1800 & 1800 & 1800 & 1800 \\
\hline
\end{tabular}

Each round lasted two minutes; and during a round ticket prices increased from 90 francs to 210 francs at a rate of 1 franc per second.

The "Own Money" rounds allowed the subjects to gamble their previous winnings with no further endowment or loan. These were excluded from all analysis presented here. Also excluded from analysis were the practice rounds. For the analysis in this paper, five 
tickets in the 010215 experiment were treated as equal to one ticket in any other experiment.

\section{Subjects and Recruiting}

The subjects were Caltech undergraduates, and for the last five experiments. Although they generally did not have experience with this particular experiment (or, presumably, the Plott, Wit, and Yang experiments performed in 1995), they generally did have previous experience as subjects in the laboratory. ${ }^{3}$

\section{Instructions and Training}

Subjects were given written instructions (which are available from the authors). These instructions were read to the subjects with the aid of some presentations on a whiteboard - items such as a drawing of an urn with states in it, the posterior probability table included above, and the earnings calculation formulae. After the instructions were read and questions were answered a practice round was opened for the subjects. This round was identical to later (non-repeat) rounds, except that no real money was involved. This round allowed subjects to familiarize themselves with the software used and the calculations required for the remaining rounds.

\section{Technology}

A purpose-built piece of software was used for all communication and transactions during the experiment. The software was used to generate the true state and private information (on the experimenter's interface); distribute the private information to subjects (through each of their screens); receive orders from subjects while the market is open; report (with each transaction by any subject) the current time in the round, the current ticket price, personal francs remaining, personal holdings of tickets, aggregate holdings of tickets (by state), and current per ticket winnings (by state); and provide a report of current round and total earnings after each round. This software was extensively tested before any experiments were performed and practice experiments were also used to ensure that everything would work smoothly.

\section{Measurement}

The results presented in the next section here are based on three important concepts: the Observed Odds of each state in the parimutuel mechanism and the Objective Winning Probability of each state. The background measure of perfection in aggregation is captured by the Aggregate Information Available.

\section{Aggregate Information Available (AIA) Model}

The AIA model represents complete information aggregation. The AIA model uses all private information (three signals for each subject) and then uses Bayes' Law to predict

\footnotetext{
${ }^{3}$ Experiment 010315 had one subject from the 010222 experiment. Experiment 010419 had one subject from 010222. Experiment 010426-2230 had one subject from 010419. Experiment 010510 had three repeat subjects (one from each of 010419, 010426-2100, and 010426-2230).
} 
the state. Achieving this measure of aggregation requires that mechanism reveal all private information.

Observed Odds of a State

The Observed Odds of a state is the fraction of tickets sold that corresponds to that state. Assuming that subjects act to maximize expected return, they will purchase tickets so that $\operatorname{Prob}(s) \times \frac{\text { Total Spending on tickets for all s }}{\text { Tickets purchased for } s}$ is constant across all states (in which they make purchases). This implies that the distribution of tickets reflects the probabilities predicted in a market of expected-return-maximizing subjects. These are the "implicit prices". The Observed Odds of a state in the mechanism are an outside observer's best estimate of the probability that a state is winning, based on publicly available market information.

Objective Winning Probabilities of a State

The Objective Winning Probability of a state given the state's Observed-Odds rank is the observed frequency that the state is actually a winning state when it has that odds rank. ${ }^{4}$ For example, of the 132 periods observed in this study, the highest-odds state was the winning state 91 times, for an Objective Winning Probability of 69\%. The exact formula is:

$$
\text { OWP }(k)=\text { Freq }[\text { Winning } \mid \text { Rank }=k]=\frac{\#(\text { Winning states with rank } \mathrm{k})}{\#(\text { Rounds })}
$$

The analysis that follows will compare these Objective Winning Probabilities to several similar benchmarks based on private information. The first considers all private signals, representing the best possible prediction of the true state. The others consider the problem faced by an individual subject - what can they derive from their individual private signals alone.

- OWP of AIA: OWP that ranks states based on the aggregate number of private signals. This Aggregate Information Available measure will generally not be knowable outside of an experimental setting, representing an unobtainable ideal prediction relying on complete revelation of all private information.

${ }^{4}$ The OWP will be calculated separately for four groups of rounds:

- Non-repeated rounds (rounds 1-4 and 17-20 in these experiments).

- 1 st round of a repeat pair (odd-numbered rounds 5-16 in these experiments).

- 2nd round of a repeat pair (even-numbered rounds 5-16 in these experiments).

- PWY non-increasing rounds (column 3 of Table 4 in Plott, Wit, and Yang, 2003). 
- OWP of InfoType3: OWP that ranks states based on the signals of subjects who receive three matching signals.

$$
\begin{aligned}
& O W P(1)=\frac{\#(\text { Subjects who receive } 3 \text { matching signals for the winnng state })}{\# \text { (Subjects with } 3 \text { matching signals of any type })} \\
& O W P(k)=\frac{1}{5} \times \frac{\#(\text { Subjects who receive } 3 \text { matching losing signals })}{\# \text { (Subjects with } 3 \text { matching signals) }} \\
& k=2,3,4,5,6
\end{aligned}
$$

- OWP of InfoType2: OWP that ranks states based on the signals of subjects who receive exactly two matching signals.

$$
\begin{aligned}
& O W P(1)=\frac{\#(\text { Subjects who receive } 2 \text { matching signals for the winning state })}{\#(\text { Subjects with } 2 \text { matching signals of any type })} \\
& O W P(2)=\frac{\#\left(\begin{array}{c}
\text { Subjects who receive } 2 \text { matching signals for losing states }) \\
\text { and } 1 \text { non }- \text { matching winning signal }
\end{array}\right)}{\# \text { (Subjects with } 2 \text { matching signals for any state })} \\
& \text { OWP }(k)=\frac{1}{4} \times \frac{\#\left(\begin{array}{c}
\text { Subjects who receive } 2 \text { matching signals for losing states }) \\
\text { and } 1 \text { non }- \text { matching losing signal }
\end{array}\right)}{\# \text { (Subjects with } 2 \text { matching signals of any type })}
\end{aligned}
$$

- OWP of InfoType1: OWP that ranks states based on the signals of subjects who receive three different signals.

$$
\begin{aligned}
& O W P(k)=\frac{1}{3} \times \frac{\#\left(\begin{array}{c}
\text { Subjects who receive } 1 \text { winning signal } \\
\text { and } 2 \text { different losing signals }
\end{array}\right)}{\# \text { (Subjects with } 3 \text { diffferent signals })} \\
& k=1,2,3 \\
& O W P(k)=\frac{1}{3} \times \frac{\# \text { (Subjects who receive } 3 \text { different losing signals) }}{\# \text { (Subjects with } 3 \text { different signals) }} \\
& k=4,5,6
\end{aligned}
$$

\section{Results}

This section contains six results. The first three address the existence of information aggregation in relation to the institutional features of the mechanism. The fourth and fifth results focus on the anatomy of the relationship between disequilibrium and the long shot bias and the final result is focused on the combination of increasing prices and repeating the application of the mechanism.

\section{RESULT 1. Information aggregation is occurring in the non-repeated increasing- price parimutuel market.}

The non-repeated increasing-price parimutuel market predicts winning states more accurately than $96 \%$ of subjects could with their private information alone. In order to see this note in Figure 1, that the state ranked most likely by the IAM market odds (probabilities) wins about $71 \%$ of the time. By contrast the state ranked most likely by those that received two identical signals (the Type 2) actually wins about $51 \%$ of the time. Thus, the market is clearly more accurate than the Type 2 individuals when 
predictions are restricted to the prediction of only one state. The predictions of Type 1 individuals are less accurate. In particular, any of the specific states of the three that are ranked most likely by the Type 1 individuals, occurs with $25 \%$ probability. Thus, the odds produced by the IAM markets are more accurate than any of the Type 1 individuals. Since the Type 2 and Type 1 individuals constitute $96 \%$ of the subjects, the IAM market is more accurate than $96 \%$ of the subjects.

Not only is the IAM more accurate than the Type 2 and Type 1 individuals when predictions are restricted to one state, the IAM market is more accurate when predictions can be expanded to include more than one state. As can be deduced from the cumulative frequencies shown in Figure 1, the state ranked second most likely by the market odds wins about $21 \%$ of the time ( $92 \%$ minus $71 \%$ ) and the state ranked third most likely by the market odds wins about $6 \%$, etc. By contrast the state ranked second by Type 2 individuals wins about $16 \%$ of the time and the state ranked third by Type 2 individuals wins about $8 \%$. Notice that the accuracy of the market is better than the Type 2 individuals when predicting the winner as one in a set of three. A similar observation holds for the Type 1 individuals and also holds for the prediction as one of a set of any size less than the universal set of six.

Computations from the data demonstrate that for Non-Repeated rounds the OWP of the market dominates the OWP of InfoType1 and InfoType2, in the sense of First Order Stochastic Dominance. ${ }^{5}$ This dominance is statistically significant. For ranks 1 thru 4 the market is better $(\mathrm{p}<1 \%)$ as it is for rank $5(\mathrm{p}<5 \%)$.

The analysis above is restricted to Type 1 and Type 2 where the market is clearly a better predictor, i.e. $96 \%$ of the subjects. In fact, the market performs about as well as the Type 3 in the Non-repeated rounds. Indeed, if the predictions are expanded to include three or more states then the IAM market is more accurate than Type 3, the best informed individuals.

Information aggregation clearly exists in the sense that an outside observer can predict better than the best informed of the individuals with private information. This property is illustrated a more detailed look at Figure 1. Shown there in the heavy line is the observed frequency with which the state ranked kth by the observed odds emerges as the winner. The state ranked first according to observed odds tends to win about $71 \%$ of the time and the state ranked second wins about $21 \%$, so the one of the first or second ranked states wins about $92 \%$ of the time. The top line is the OWP of the AIA, which represents the actual collection of all information held by individuals. The state ranked first by AIA is the winner about $98 \%$ of the time. This difference with the actual frequencies reveals that the information aggregation in the Non Repeat rounds is not perfect. However, comparisons with the various information types reveal how good the aggregation is relative to the most informed. We see the first ranked state by Type 3 occurring with

\footnotetext{
${ }^{5}$ For Info Type i $(\mathrm{i}=1,2)$ and for each rank k, Observed Freq[Winning $\mid$ Rank according to Odds less than or equal to $\mathrm{k}$ ] is larger than Freq[Winning | Rank according to InfoType $\mathrm{i}$ is less than or equal to $\mathrm{k}]$.
} 
about $90 \%$ of the time, which is more than the $71 \%$ of the market but not statistically significantly so. However, when considering the top two ranked states the market and the Type 3 have about equal accuracy (Type 3 is 92.4\% and the market is 91.7\%). AIA is a significantly better predictor of winners than observed odds ( $\mathrm{p}<1 \%$ for rank 1 ). ${ }^{6}$ Except for those mentioned otherwise, no other differences in the figure are significant at the $5 \%$ level.

Another method to measure information aggregation produces similar results. A chisquared test for goodness-of-fit can be used to test how well the observed odds or each InfoType is able to predict the observed winning states. Under this test, the observed odds, AIA, and InfoType3 predict the winning states adequately, while InfoType1 and InfoType 2 are both bad predictors of the winning state $(\mathrm{p}<1 \%)$.

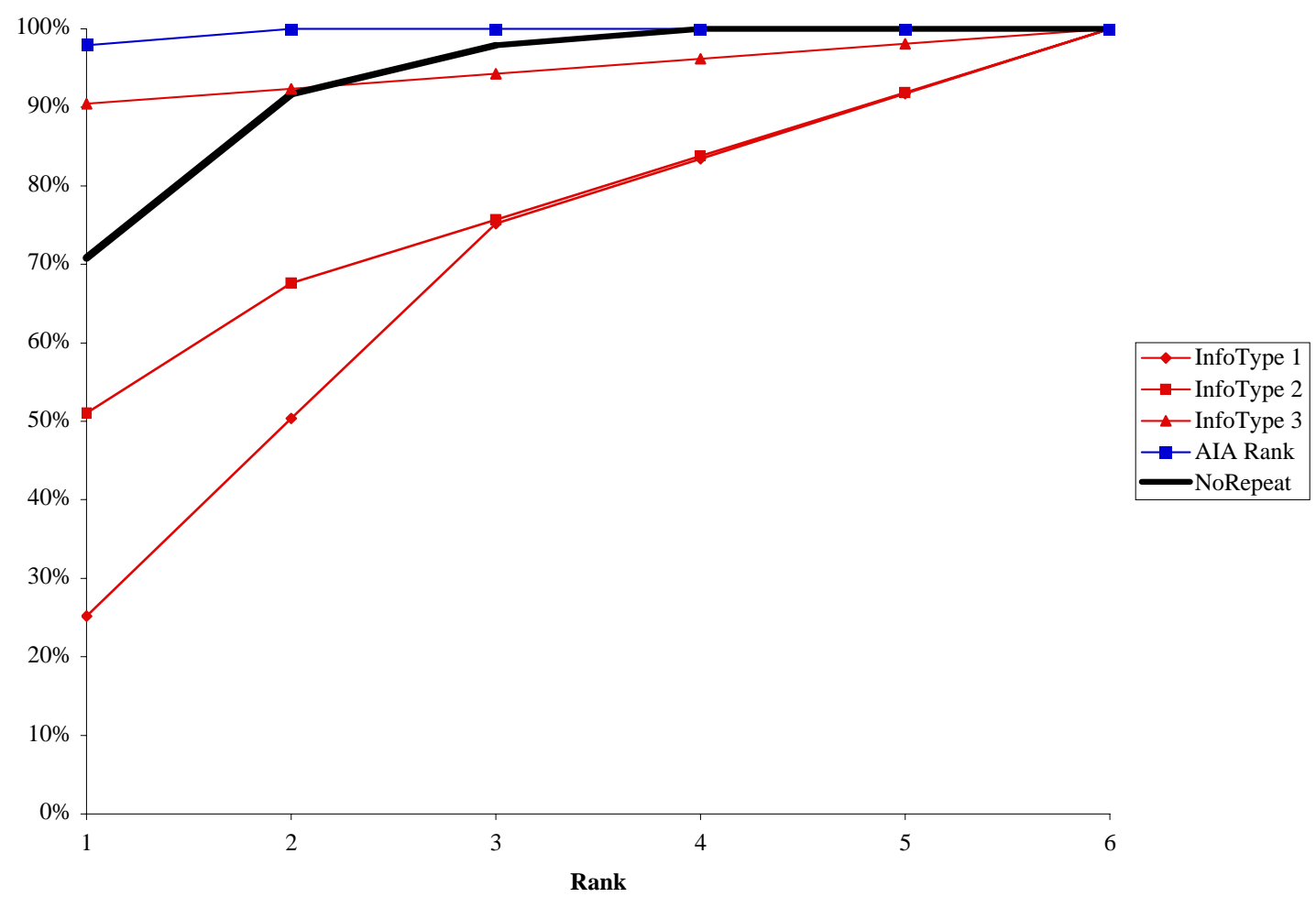

Figure 1: Non-repeated increasing-price parimutuel markets predict winners better than most subjects, matching the few to receive three matching signals.

\section{RESULT 2. Information aggregation is occurring in the repeated increasing-price parimutuel market.}

The repeated increasing-price parimutuel market predicts winning states more accurately than $96 \%$ of subjects could with their private information alone, and closely matches the remaining subjects. The reasoning is the same as used in the support for Result 1 . The OWP for both 1st and 2nd rounds of a repeat pair dominate the OWP of InfoType1 and

\footnotetext{
${ }^{6}$ Using a Fisher-Irwin test, an exact hypergeometric test, as in Ross (1987, 232-233).
} 
InfoType2 in these rounds, in the sense of First Order Stochastic Dominance. The data are shown in Figure 2. The OWP for the most likely outcome as predicted by the IAM are about $65 \%$ for the first round of a repeated pair and about $70 \%$ for the second round of the repeated power. ${ }^{7}$ The 1st round of a repeat pair performs about as well as InfoType3. The 2 nd round of a repeat pair nearly matches InfoType3 for rank 1 (71.4\% versus $72.2 \%$ winners), and dominates for all lower ranks. ${ }^{8}$ The 1 st round of a repeat pair is dominated by AIA, the unattainable benchmark. The 2nd round of a repeat pair actually performs better than AIA at rank 3 - all 42 winning states were ranked 1, 2, or 3 by observed odds, while only 41 of those states were ranked 1, 2, or 3 by the number of signals, Figure 2 .

Observed odds in the 1st or 2nd round of a repeat pair are a significantly better predictor of winners than InfoType1 or InfoType2 ( $<<1 \%$ for ranks $1-4, p<5 \%$ for rank 5 ). AIA is a significantly better predictor of winners than 1 st round observed odds ( $\mathrm{p}<5 \%$ for ranks 1 and 2). No other differences are significant at the $5 \%$ level. That is, if the prediction is made from the second round of a repeated pair there is no statistical difference between the accuracy of predictions of AIA and the odds produced by the IAM.

As in the previous result, a chi-squared test for goodness-of-fit finds that the predictions of InfoType1 $(\mathrm{p}<1 \%)$ and InfoType2 $(\mathrm{p}<5 \%)$ poorly match the observed winning states. The observed odds, AIA, and InfoType3 predictions pass this test, again suggesting that the repeated parimutuel market is making predictions as good as the generally unknowable benchmark using all private information.

\footnotetext{
${ }^{7}$ One might wonder why the OWP of about $65 \%$ for the first round of a repeated pair are lower than the OWP for the non repeated rounds shown in Figure 1. The answer lies in the lower OWP of AIA in the first rounds of the repeated pairs, which demonstrates that the information to be aggregated was worse for the repeated pair rounds. It was just the randomness of the draws happened to produce less information to be aggregated.

${ }^{8}$ In the 42 repeat pairs, the winning state had the highest observed odds in the 2nd round in 30 cases $(30 / 42=71.4 \%)$. Of the 18 subjects receiving three matching signals in these 42 rounds, 13 received correct signals of the winning state $(13 / 18=72.2 \%)$.
} 


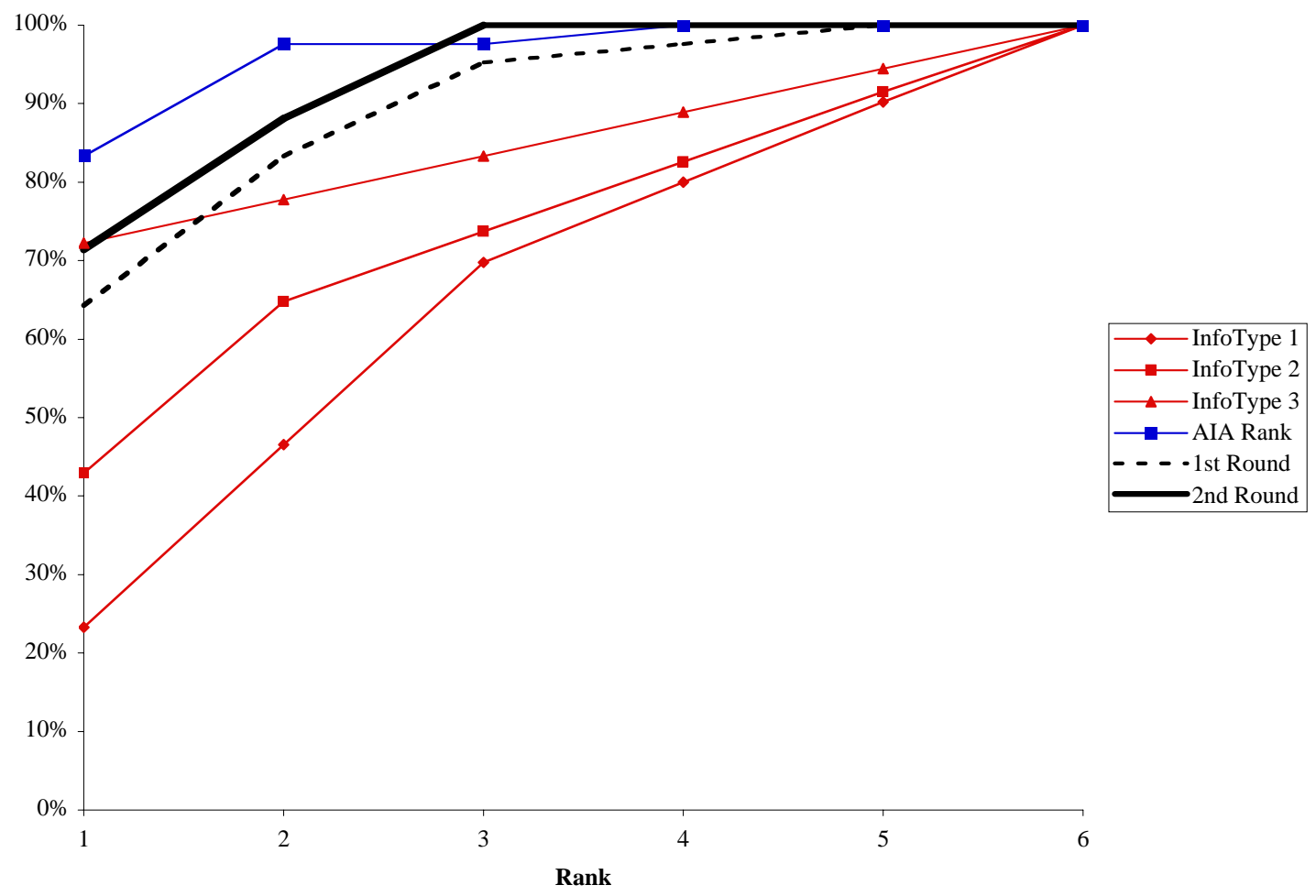

Figure 2: Repeated increasing-price parimutuel markets predict winners better than most subjects, matching the predictive capacity of the few to receive three matching signals and also matching the aggregate information available.

\section{RESULT 3. Repeated and non-repeated increasing-price parimutuel markets perform better than previously-tested parimutuel markets.}

Both repeated and non-repeated parimutuel markets predict winning states more accurately than the constant-price parimutuel market tested by Plott, Wit, and Yang (2003). ${ }^{9}$ The OWP for non-repeated rounds, the 1 st round of a repeat pair, and the 2 nd round of a repeat pair dominate the OWP of the Plott, Wit, and Yang markets, in the sense of First Order Stochastic Dominance. See Figure 3.

Observed odds in the 2nd round of a repeat pair are a significantly better predictor of winners than PWY markets ( $<<5 \%$ for rank 3). No other differences are significant at the $5 \%$ level.

\footnotetext{
${ }^{9}$ Plott, Wit, and Yang consider two information environments. Their PIC setting exactly matches the setting here, and only those rounds are used for comparison.
} 


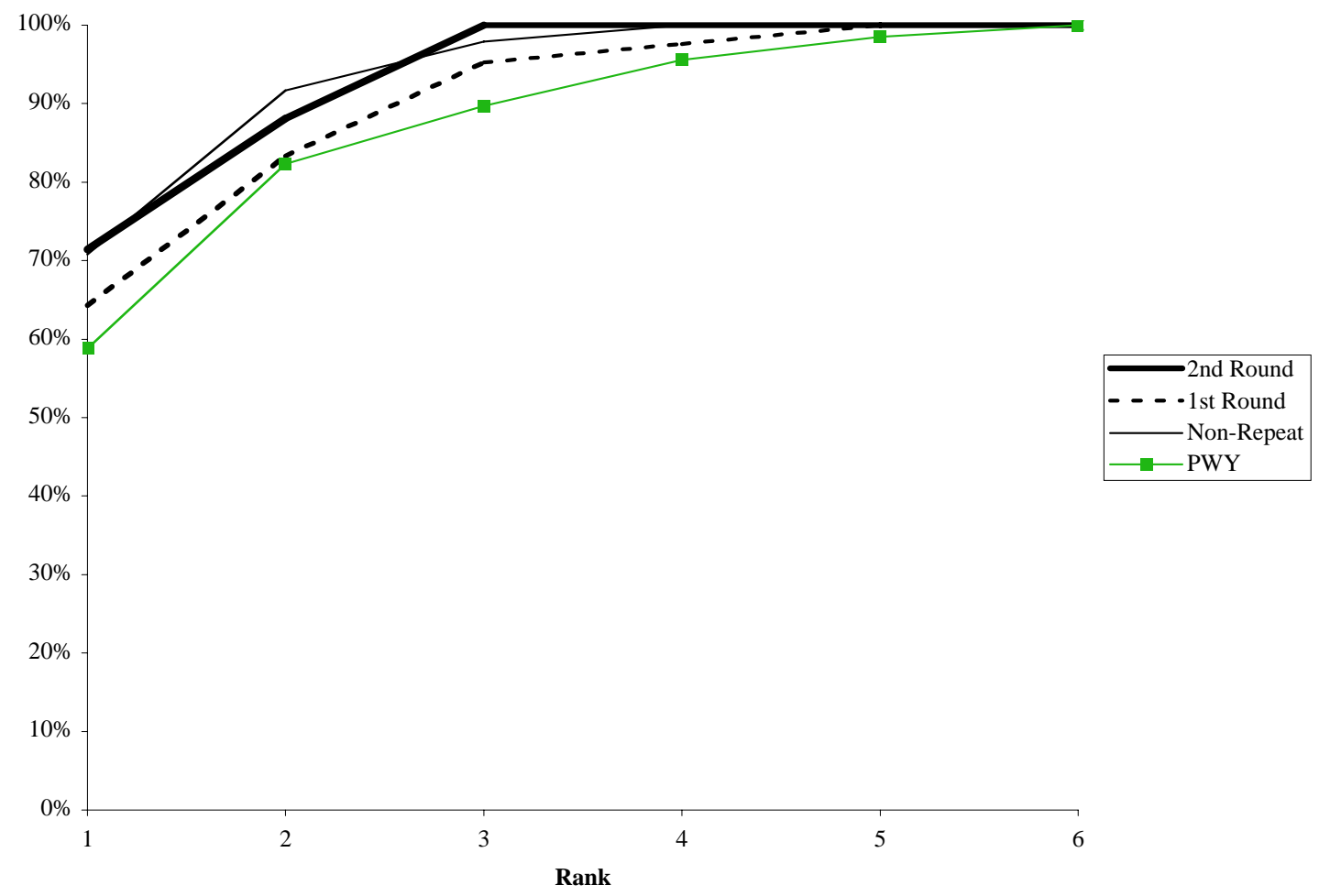

Figure 3: Repeated and non-repeated increasing-price parimutuel markets predict winners better than markets studied by Plott, Wit, and Yang (2003).

\section{RESULT 4. Non-repeated increasing-price parimutuel markets exhibit a long-shot bias.}

Non-repeated parimutuel markets demonstrate a long-shot bias, where the observed odds exceed the objective winning probability for unlikely states. This means that subjects are wagering too much money on unlikely events and not enough on likely events, as measured by the actual frequency of winners. For this analysis we have pooled the data from the non-repeated sessions and the first period of the repeated sessions. The patterns of these data sets are very similar but the pooling allows an increase in the sample size needed for statistical significance. As reflected in the table below, the observed odds for the two highest-odds (and objectively most likely) states are too low (respectively 0.628 and 0.173 ) as compared to actual frequencies of 0.678 and 0.20 ) and the odds for the three most unlikely states are too high (respectively $0.051,0.032,0.021$ as compared with $0.022,0.011,0.000$ ). The result is the so called "long shot bias" in which there are significantly too many tickets for the 4th, 5th, and 6th state being bought, relative to the objective frequency of winning ( $<<5 \%$ one-tailed). 
Long-Shot Bias in Non-Repeated or First of a Repeat Pair Rounds

\begin{tabular}{cccc}
$\begin{array}{c}\text { Rank of } \\
\text { Observed } \\
\text { Odds }\end{array}$ & $\begin{array}{c}\text { Average } \\
\text { Observed } \\
\text { Odds at rank }\end{array}$ & $\begin{array}{c}\text { Objective } \\
\text { Frequency of } \\
\text { winners at } \\
\text { rank (OWP) }\end{array}$ & $\begin{array}{c}\text { t-statistic of } \\
\text { (Odds-OWP) }\end{array}$ \\
\hline 1st & 0.628 & $0.678(61 / 90)$ & -0.96 \\
\hline 2nd & 0.173 & $0.200(18 / 90)$ & -0.62 \\
\hline 3rd & 0.095 & $0.089(8 / 90)$ & +0.19 \\
\hline 4th & 0.051 & $0.022(2 / 90)$ & +1.82 \\
\hline 5th & 0.032 & $0.011(1 / 90)$ & +1.85 \\
\hline 6th & 0.021 & $0.000(0 / 90)$ & $>1.94$ \\
\hline
\end{tabular}

The pattern of bias in these rounds is consistent with that reported by Plott, Wit, and Yang (2003, Table 4). They also found that there was too little betting on the two mostlikely states while the four least-likely states were over-represented.

\section{RESULT 5. Repeated increasing-price parimutuel markets do not exhibit a long-shot bias.}

The second round of a repeated pair of parimutuel markets does not demonstrate a longshot bias. Subjects are wagering slightly too much money on the most likely event and not enough on mid-ranked events, relative to the objective probability of winning as reflected in the table below. None of these differences are statistically significant even at $\mathrm{p}=10 \%$.

\begin{tabular}{cccc}
\hline \multicolumn{4}{c}{ No Long-Shot Bias in the Second Round of a Repeat Pair } \\
$\begin{array}{c}\text { Rank of } \\
\text { Observed } \\
\text { Odds }\end{array}$ & $\begin{array}{c}\text { Average } \\
\text { Observed } \\
\text { Odds at rank }\end{array}$ & $\begin{array}{c}\text { Objective } \\
\text { Frequency of } \\
\text { winners at } \\
\text { rank (OWP) }\end{array}$ & $\begin{array}{c}\text { t-statistic of } \\
\text { (Odds-OWP) }\end{array}$ \\
\hline 1st & 0.728 & $0.714(30 / 42)$ & +0.13 \\
\hline 2nd & 0.148 & $0.167(7 / 42)$ & -0.32 \\
\hline 3rd & 0.069 & $0.119(5 / 42)$ & -1.00 \\
\hline 4th & 0.030 & $0.000(0 / 42)$ & $>1.26$ \\
\hline 5th & 0.017 & $0.000(0 / 42)$ & $>0.73$ \\
\hline 6th & 0.013 & $0.000(0 / 42)$ & $>0.55$ \\
\hline
\end{tabular}

RESULT 6. Repeating rounds and increasing prices improve the accuracy of betting in the parimutuel market.

The observed odds of the highest-rank event are higher, and the odds of other events are lower, in repeated parimutuel markets than in non-repeated increasing markets. This same pattern holds when comparing either type of increasing-price market to Plott, Wit, and Yang's constant-price market. The differences between the repeated and nonrepeated markets are statistically significant $(\mathrm{p}<1 \%$ for all except the second rank, with $\mathrm{p}<10 \%$ ). The repeated, increasing-price parimutuel is also a significant improvement 
over the constant-price parimutuel (PWY), with $\mathrm{p}<1 \%$ for all states. The non-repeated parimutuel is significantly better for the 1 st, 2 nd, and 6th $\operatorname{rank}(\mathrm{p}<1 \%) .{ }^{10}$

\begin{tabular}{ccccc}
\hline \multicolumn{5}{c}{ Market refinements significantly improve market performance } \\
\hline Rank of & $\begin{array}{c}\text { t-statistic of } \\
\text { (2nd Round }\end{array}$ & $\begin{array}{c}\text { t-statistic of } \\
\text { (2nd Round }\end{array}$ & $\begin{array}{c}\text { t-statistic of } \\
\text { (2nd Round }\end{array}$ & $\begin{array}{c}\text { t-statistic of } \\
\text { (No Repeat }\end{array}$ \\
$\begin{array}{c}\text { Observed } \\
\text { Odds }\end{array}$ & $\begin{array}{c}\text { 1st Round } \\
\text { Odds) }\end{array}$ & $\begin{array}{c}\text { No Repeat } \\
\text { Odds) }\end{array}$ & $\begin{array}{c}\text { - PWY } \\
\text { Odds) }\end{array}$ & $\begin{array}{c}\text { - PWY } \\
\text { Odds) }\end{array}$ \\
\hline 1st & +2.15 & +3.37 & +5.39 & +2.10 \\
\hline 2nd & -1.21 & -1.33 & -3.49 & -3.16 \\
\hline 3rd & -1.94 & -2.76 & -3.55 & -0.96 \\
\hline 4th & -2.75 & -4.63 & -5.82 & -1.19 \\
\hline 5th & -2.54 & -5.30 & -6.96 & -1.66 \\
\hline 6th & -1.44 & -4.02 & -6.06 & -2.04 \\
\hline
\end{tabular}

\section{Summary and Conclusions}

This paper picks up a challenge offered by Plott, Wit, and Yang (2003), who discovered that an IAM designed along the lines of parimutuel betting systems can aggregate information. In spite of the success of the mechanism they studied, PWY reported several aspect of strategic behavior that seemed to work against any success and offered a challenge to design modifications for improvement. Both theory and data call attention to two features. First, within the PWY mechanism, there exists no incentive to place bets early. Secondly, incentives exist to bluff and mislead. Third, much disequilibrium behavior transpired that manifested itself in the final odds and thus the predictions.

The research reported here studies a mechanism of increasing prices for tickets designed to address the first problem of delay. The increasing prices increase the cost of delay. A second feature involves a replication of the mechanism after a first round of predictions are known thereby attempting to remove the impact of disequilibrium decisions. The results demonstrate that the two features when incorporated into a newly structured IAM substantially improve the prediction capability of the mechanism. Furthermore, the research is able to establish a connection between the institutional feature of the IAM design and the particular types of biases that can exist.

The principle results reside in Result 2, which claims that the IAM actually aggregates information. The prediction properties do not result from the actions of a single knowledgeable individual. The information contained in the odds is better than the information held by any single individual. The property is seen in the fact that the predictions of the IAM when applied to more than just predicting one state as the winner.

${ }^{10}$ Plott, Wit, and Yang (2003) do not report the standard error for their estimates of average implicit price (observed odds) given rank. Since their sample is slightly larger than ours, their errors should be smaller. The t-stats are calculated based on the standard errors for the 2nd-1st and 2nd-No comparisons. 
That is, at this task the IAM is better than the best informed of individuals and therefore reflects more information than is held by the best informed of the individuals.

In addition to establishing the information aggregation properties of the IAM and the role of institutions in forming its accuracy, the results resolve a long standing debate about the nature of a long shot bias frequently observed in parimutuel betting systems. The long shot bias in the experimental parimutuel IAMs is due to disequilibrium choices and not to a property of psychological limitations of some sort.

Although the parimutuel market does aggregate information, it does not do so perfectly. Even though the information aggregation is strong, mirages and bubbles still occur from time to time. How they might be removed and the IAM improved accordingly remains a challenge. 


\section{References}

Camerer, Colin F.; 1998. "Can Asset Markets Be Manipulated? A Field Experiment with Racetrack Betting." Journal of Political Economy, June, 457-82.

Chen, K. Y., and C. Plott; 2002; "Information Aggregation Mechanisms: Concept, Design and Implementation for a Sales Forecasting Problem”; Social Science Working Paper 1131, California Institute of Technology.

Plott, C. 2000; “Markets as Information Gathering Tools”; Southern Economic Journal 67(1) (2000):1-15.

Plott, C., J. Wit, and W. Yang; 2003; "Parimutuel betting markets as information aggregation devices: experimental results"; Economic Theory 22, 311-351.

Plott, C., and S. Sunder; 1982; "Efficiency of Experimental Security Markets with insider Information: An Application of Rational Expectations Models,”; Journal of Political Economy 90 (August 1982):663-698.

Plott, C., and S. Sunder; 1988; "Rational Expectations and the Aggregation of Diverse Information in Laboratory Security Markets"; Econometrica 56:1085-1118.

Ross, S.; 1987; Introduction to Probability and Statistics for Engineers and Scientists; John Wiley \& Sons.

Thaler, Richard and William T. Ziemba, “Anomalies: Parimutuel Betting Markets: Racetracks and Lotteries”, Journal of Economic Perspectives, Vol.2, No.2 (Spring, 1988), pp.161-174. 